BULL. AUSTRAL. MATH. SOC.

VOL. $27(1983), 215-219$.

\title{
COMPLETE HYPERSURFACE OF NON-POSITIVE RICCI CURVATURE
}

\author{
Pui - Fai Leung
}

\begin{abstract}
We conjecture that a complete hypersurface of non-positive Ricci curvature in the Euclidean space must be unbounded. We prove this under the additional assumption that all sectional curvatures of the hypersurface are bounded away from negative infinity.
\end{abstract}

\section{Introduction}

In this note we shall consider a complete hypersurface $M^{n}$ in an Euclidean space $\mathbb{R}^{n+l}$. We shall consider the case when the Ricci curvature of $M$ is non-positive. We first observe that it follows from the Gauss equation that any minimal hypersurface belongs to our class. of course there are hypersurfaces with non-positive Ricci curvature which are not minimal. For the case of minimal hypersurfaces it has been conjectured for a long time that they cannot be bounded. It is well-known that minimal hypersurfaces cannot be compact and it is also well-known that even the larger class of hypersurfaces with non-positive Ricci curvature cannot have compact members either, therefore it seems likely that the following stronger conjecture may be true.

CONJECTURE. Any complete hypersurface with non-positive Ricci curvature in the Euclidean space must be unbounded.

The example of a flat torus $S^{1} \times S^{l}$ in $\mathbb{R}^{2} \times \mathbb{R}^{2}$ shows that we cannot relax the codimension in the above conjecture.

Received 18 October 1982. 
In the following we shall show that under the additional assumption that the sectional curvatures of the hypersurface are bounded away from $-\infty$, the conjecture is true. Our proof is a direct application of an important theorem due to Omori [1] and an algebraic result on bilinear forms due to Otsuki [2].

\section{Introduction}

In this section we shall state the theorem of Omori and that of Otsuki to be used in the next section.

Since a smooth function $f$ attains a maximum on a compact manifold (at a point $p$ say) we have $\operatorname{grad} f(p)=0$ and Hess $f(X, X) \leq 0$ for any unit vector $X$ in the tangent space at $p$. The theorem of Omori is a generalization of this phenomenon.

THEOREM (Omori [1]). Let $M$ be a complete and connected Riemannian manifold whose sectional curvatures are bounded caway from $-\infty$. Let $f$ be a smooth and bounded fronction on $M$. Then, for any $\varepsilon>0$, there is a point $p \in M$ such that $\|\operatorname{grad} f(p)\|<\varepsilon$ and Hess $f(X, X)<\varepsilon$ for any unit vector $X \in T M_{p}$.

Next we consider a symmetric bilinear form $B: \mathbb{R}^{n} \times \mathbb{R}^{n}+\mathbb{R}^{p}$. Let $s^{n-1}$ denote the unit sphere in $\mathbb{R}^{n}$. Consider the function $\phi: S^{n-1} \rightarrow \mathbb{R}$ defined by $\phi(X)=\|B(X, X)\|^{2}$. Clearly $\phi$ is smooth and since $s^{n-1}$ is compact $\phi$ attains a minimum at $X_{0}$. We shall consider the linear transformation $B\left(x_{0}, \cdot\right): \mathbb{R}^{n} \rightarrow \mathbb{R}^{p}$.

THEOREM ([2], Chapter 1], Lemma 1). Suppose $B\left(x_{0}, x_{0}\right) \neq 0$. Then (i) $x_{0} \perp \operatorname{Ker} B\left(x_{0}, \cdot\right)$,

(ii) for any $Y \in \operatorname{Ker} B\left(x_{0}, \cdot\right)$, we have

$$
\left\langle B\left(x_{0}, X_{0}\right), B(y, Y)\right\rangle \geq\left\|B\left(x_{0}, x_{0}\right)\right\|^{2} .
$$




\section{The main result}

Consider now a complete hypersurface $M^{n}$ in the Euclidean space $\mathbf{R}^{n+1}$. We shall denote the connection on $M^{n}$ by $\nabla$ and the connection on $\mathbb{R}^{n+1}$ by $\tilde{\nabla}$. The second fundamental form $B$ is a symmetric bilinear form on $T M \times T M$ into $N M$ (the normal bundle) given by

$$
\tilde{\nabla}_{X} Y=\nabla_{X} Y+B(X, Y)
$$

where $X, Y \in T M$. For a pair of orthogonal unit vectors $X, Y \in T M$, we shall denote by $R(X, Y, X, Y)$ the sectional curvature corresponding to the plane containing $X$ and $Y$. We have the Gauss equation

$$
R(X, Y, X, Y)=\langle B(X, X), B(Y, Y)\rangle-\|B(X, Y)\|^{2} .
$$

For any unit vector $X \in T M$, the Ricci curvature in that direction is given by

$$
\operatorname{Ric}(X, X)=\sum_{i=1}^{n-1} R\left(X, Y_{i}, X, Y_{i}\right) \text {, }
$$

where $\left\{X, Y_{1}, \ldots, Y_{n-1}\right\}$ form an orthonormal basis of $T M$.

Now suppose that $M$ is bounded. So $M$ lies inside a ball of radius $r$ say. We consider the function $f$ on $M$ defined by $f(x)=(x, x)$ where $x$ stands for the position vector of $M$. Clearly $f(x) \leq r^{2}$ and so is bounded.

Now take any point $p \in M$ and any unit vector $V \in T M_{p}$. We shall now compute Hess $f(V, V)$. We first recall that $\tilde{\nabla}_{V} x=V$ when $x$ is the position vector. We have

$$
\text { Hess } \begin{aligned}
f(V, V) & =V V(f)-\nabla_{V} V(f) \\
& =V V\langle x, x\rangle-\nabla_{V} V\langle x, x\rangle \\
& =2 V\langle V, x\rangle-2\left\langle\nabla_{V} V, x\right\rangle \\
& =2\left\langle\tilde{\nabla}_{V} V, x\right\rangle+2\langle V, V\rangle-2\left\langle\nabla_{V} V, x\right\rangle \\
& =2\langle(B(V, V), x\rangle+2 .
\end{aligned}
$$

Now for any positive integer $m$, we have by Omori's theorem a point $p \in M$ 
so that Hess $f(V, V)<2 / m$ for all unit vectors $V \in T M_{p}$. Therefore we have

$$
\begin{aligned}
\|B(V, V)\| & \geq \frac{1}{r}\|x\|\|B(V, V)\| \\
& \geq \frac{-1}{r}\langle B(V, V), x\rangle \\
& =\frac{1}{2 r}(2-\text { Hess } f(V, V)) \\
& >\frac{1}{r}(1-(1 / m))
\end{aligned}
$$

and so $B(V, V) \neq 0$.

Now we take $X_{0}$ so that $\left\|B\left(X_{0}, X_{0}\right)\right\|^{2}$ is the minimum of $\|B(V, V)\|^{2}$ for all units $V \in T M_{p}$. From above $B\left(X_{0}, X_{0}\right) \neq 0$ and since $\operatorname{dim} \operatorname{Ker} B\left(X_{0},-\right) \geq n-1$ we therefore have $\operatorname{dim} \operatorname{Ker} B\left(X_{0}, \cdot\right)=n-1$. Take $Y_{1}, \ldots, Y_{n-1}$ to be an orthonormal basis for $\operatorname{Ker} B\left(x_{0}, \cdot\right)$. By $(i)$ in Otsuki's theorem, we have an orthonormal basis $X_{0}, Y_{1}, \ldots, Y_{n-1}$ for $T M_{p}$. It therefore follows from the Gauss equation and (ii) in Otsuki's theorem that

$$
\begin{aligned}
\operatorname{Ric}\left(x_{0}, X_{0}\right) & =\sum_{i=1}^{n-1} R\left(x_{0}, y_{i}, x_{0}, y_{i}\right) \\
& =\sum_{i=1}^{n-1}\left(B\left(x_{0}, x_{0}\right), B\left(y_{i}, y_{i}\right)\right) \\
& \geq \sum_{i=1}^{n-1}\left\|B\left(x_{0}, x_{0}\right)\right\|^{2} \\
& >\frac{n-1}{r^{2}}(1-(1 / m))^{2} .
\end{aligned}
$$

Hence letting $m \rightarrow \infty$, we obtain the following.

THEOREM. Let $M^{n}$ be a complete hypersurface in $\mathbb{R}^{n+1}$ such that all sectional curvatures on $M$ are bounded caway from $-\infty$. If $M$ is contained in a ball of radius $r$, then 


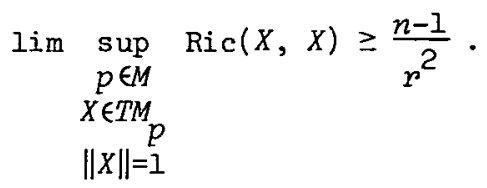

From this we have the following partial answer to our conjecture.

COROLLARY. Let $M^{n}$ be a complete hypersurface in $\mathbb{R}^{n+1}$ such that all sectional curvatures on $M$ are bounded coway from $-\infty$. If $M$ has non-positive Ricci curvature, then $M$ is unbounded.

\section{References}

[1] Hideki Omori, "Isometric immersions of Riemannian manifolds", J. Math. Soc. Japan 19 (1967), 205-214.

[2] Michael Spivak, A comprehensive introduction to differential geometry, Volume 5 (Publish or Perish, Boston, Massachusetts, 1975).

Department of Mathematics,

National University of Singapore,

Kent Ridge,

Singapore 0511 . 